\title{
Erratum to: $N$-Glycosylation profiling of recombinant mouse extracellular superoxide dismutase produced in Chinese hamster ovary cells
}

\author{
Hiroaki Korekane • Atsuko Korekane • Yoshiki Yamaguchi • Masaki Kato • \\ Yasuhide Miyamoto • Akio Matsumoto • Tomoko Hasegawa • Keiichiro Suzuki • \\ Naoyuki Taniguchi $\cdot$ Tomomi Ookawara
}

Published online: 14 June 2011

(C) Springer Science+Business Media, LLC 2011

\section{Erratum to: Glycoconj J (2011) 28:183-196 \\ DOI 10.1007/s10719-011-9333-6}

The addition of grant acknowledgement below was inadvertently missed at proof stage.

Hiroaki Korekane and Atsuko Korekane contributed equally to this work.

The online version of the original article can be found at http://dx.doi. org/10.1007/s10719-011-9333-6.

H. Korekane $\cdot$ T. Hasegawa $\cdot$ N. Taniguchi

Department of Disease Glycomics (Seikagaku Corporation), The Institute of Scientific and Industrial Research, Osaka University,

8-1 Mihogaoka, Ibaraki, Osaka 567-0047, Japan

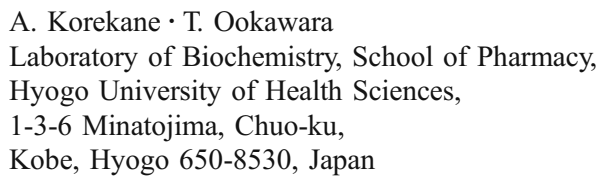

H. Korekane $\cdot$ Y. Yamaguchi $\cdot$ M. Kato $\cdot$ N. Taniguchi

Systems Glycobiology Research Group, Chemical Biology

Department, Advanced Science Institute,

RIKEN, 2-1 Hirosawa,

Wako, Saitama 351-0198, Japan

\section{Y. Miyamoto}

Department of Immunology,

Osaka Medical Center for Cancer and Cardiovascular Diseases,

1-3-2 Nakamichi, Higashinari-ku, Osaka 537-8511, Japan

\section{A. Matsumoto}

Department of Pharmacology,

Chiba University Graduate School of Medicine,

1-8-1 Inohana, Chuo-ku, Chiba 260-8670, Japan

\section{Acknowledgements section:}

This work was also supported by the grant in relation to "Validation of the treatment protocol using glycosaminoglycans for COPD exacerbation in vitro and Exploring biomarkers associating COPD exacerbation" from National Institute of Biomedical Innovation (NIBIO) in Japan.

\section{K. Suzuki}

Department of Biochemistry, Hyogo College of Medicine, 1-1 Mukogawa-cho, Nishinomiya, Hyogo 663-8501, Japan

\footnotetext{
N. Taniguchi $(\bowtie)$

Department of Disease Glycomics (Seikagaku Corporation), The Institute of Scientific and Industrial Research, Osaka University, 8-1 Mihogaoka, Ibaraki, Osaka 567-0047, Japan e-mail: tani52@wd5.so-net.ne.jp
}

\author{
N. Taniguchi \\ Systems Glycobiology Research Group, Chemical Biology \\ Department, Advanced Science Institute, \\ RIKEN, 2-1 Hirosawa, Wako, Saitama 351-0198, Japan
}

T. Ookawara $(\bowtie)$

Laboratory of Biochemistry, School of Pharmacy,

Hyogo University of Health Sciences,

1-3-6 Minatojima, Chuo-ku, Kobe, Hyogo 650-8530, Japan

e-mail: biochook@huhs.ac.jp 